\title{
A Sub-Millimeter solenoid Device for Trapping Paramagnetic Microbeads
}

\author{
L. D. Garcia, L. C. Cheung, J. C. Mikkelsen, Jr., J. G. \\ Santiago, A. F. Bernhardt, V. Malba
}

This article was submitted to 2001 American Society of Mechanical Engineers International Mechanical Engineering Congress and Exposition, New York, NY, November 11-16, 2001

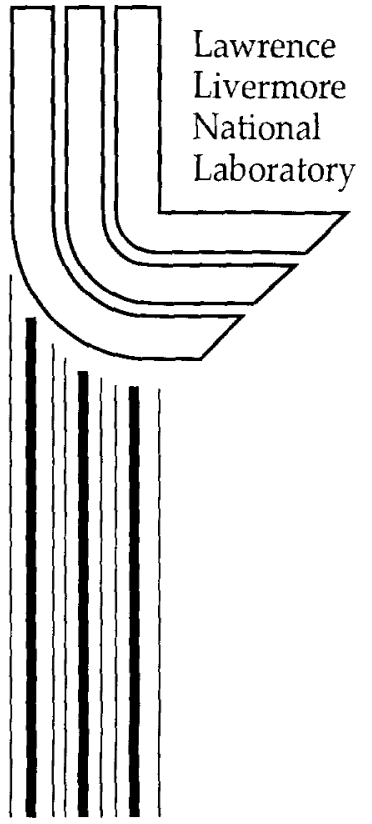

\section{August 1, 2001}




\section{DISCLAIMER}

This document was prepared as an account of work sponsored by an agency of the United States Government. Neither the United States Government nor the University of California nor any of their employees, makes any warranty, express or implied, or assumes any legal liability or responsibility for the accuracy, completeness, or usefulness of any information, apparatus, product, or process disclosed, or represents that its use would not infringe privately owned rights. Reference herein to any specific commercial product, process, or service by trade name, trademark, manufacturer, or otherwise, does not necessarily constitute or imply its endorsement, recommendation, or favoring by the United States Government or the University of California. The views and opinions of authors expressed herein do not necessarily state or reflect those of the United States Government or the University of California, and shall not be used for advertising or product endorsement purposes.

This is a preprint of a paper intended for publication in a journal or proceedings. Since changes may be made before publication, this preprint is made available with the understanding that it will not be cited or reproduced without the permission of the author.

This work was performed under the auspices of the United States Department of Energy by the University of California, Lawrence Livermore National Laboratory under contract No. W-7405-Eng-48.

This report has been reproduced directly from the best available copy.

Available electronically at http://www.doc.gov/bridge

Available for a processing fee to U.S. Department of Energy

And its contractors in paper from

U.S. Department of Energy

Office of Scientific and Technical Information

P.O. Box 62

Oak Ridge, TN 37831-0062

Telephone: (865) 576-8401

Facsimile: (865) 576-5728

E-mail: reports@adonis.osti.gov

Available for the sale to the public from

U.S. Department of Commerce

National Technical Information Service

5285 Port Royal Road

Springfield, VA 22161

Telephone: (800) 553-6847

Facsimile: (703) 605-6900

E-mail: orders@ntis.fedworld.gov

Online ordering: http://www.ntis.gov/ordering.htm

\section{OR}

Lawrence Livermore National Laboratory

Technical Information Department's Digital Library

http://www.llnl.gov/tid/Library.html 


\title{
A SUB-MILLIMETER SOLENOID DEVICE FOR TRAPPING PARAMAGNETIC MICROBEADS
}

\author{
Luis D. García, Lawrence C. Cheung, James C. Mikkelsen Jr., Juan G. Santiago \\ Department of Mechanical Engineering, Stanford University \\ BIdg. 500, Room 500Y, Stanford, CA 94305 USA \\ phone: 650.723 .4881 fax: 650.723.7657 \\ Idg@Stanford.EDU
}

Anthony F. Bernhardt, Vincent Malba

Lawrence Livermore National Laboratory

P.O. Box 808, L-286

Livermore, CA 94551 USA

\begin{abstract}
We present the design and preliminary evaluation of a paramagnetic microsphere trapping and separation device consisting of a copper solenoid wrapped around a $1.3 \mathrm{~mm}$ diameter glass capillary. The magnetization and subsequent dipole-dipole interaction of paramagnetic spheres under an applied magnetic field results in the formation of bead chains that persist and grow under the applied field, but quickly disperse upon field removal. The chaining of paramagnetic spheres is important to the design of magnetic-based separation devices because the viscous-drag-limited velocities of chains are typically several times larger than that of individual particles. We have performed a set of experiments designed to evaluate the performance of a sub-millimeter solenoid device including measurements of the temperature versus field strength of the device, observations of the controlled chain formation process, and preliminary observations regarding the maximum flow rate over which the bead chains can be held in place by magnetic forces. These results are applicable to the design and characterization of magnetically induced microsphere trapping and separation systems which use pressure driven flow.
\end{abstract}

\section{NOMENCLATURE}

Symbol Definition

$B \quad$ Magnetic field

$B_{r} \quad$ Magnetic field (radial)

$B_{z} \quad$ Magnetic field (axial)

I Solenoid coil current

Solenoid coil length

Number of solenoid coil turns

Fluid-ambient temperature difference

Particle volume

\begin{tabular}{cl}
$d$ & Particle diameter \\
$g$ & Gravitational acceleration \\
$h_{e f f}$ & Effective heat transfer coefficient \\
$r_{0}$ & Outer capillary radius \\
$r_{i}$ & Inner capillary radius \\
$v$ & Particle velocity \\
$v_{s}$ & Particle settling velocity \\
$z^{*}$ & Non-dimensional axial coordinate \\
$\Delta \chi$ & Magnetic susceptibility difference \\
$\Delta \rho$ & Particle-fluid density difference \\
$\eta^{\prime}$ & Solenoid coil pitch \\
$\mu_{0}$ & Dynamic viscosity \\
\hline
\end{tabular}

$$
\begin{gathered}
\mathrm{m} \\
\mathrm{m} / \mathrm{s}^{2} \\
\mathrm{~W} /\left(\mathrm{m}^{2} \mathrm{~K}\right) \\
\mathrm{m} \\
\mathrm{m} \\
\mathrm{m} / \mathrm{s} \\
\mathrm{m} / \mathrm{s} \\
\mathrm{none} \\
\text { none } \\
\mathrm{kg} / \mathrm{m}^{3} \\
\mathrm{degrees} \\
\mathrm{kg} /\left(\mathrm{m} \mathrm{s}^{\mathrm{s}}\right) \\
\mathrm{N} / \mathrm{A}^{2}
\end{gathered}
$$

\section{INTRODUCTION}

Investigation of paramagnetic microsphere dynamics in aqueous solutions is motivated by the development of bioanalytical systems that handle, concentrate, and separate chemical species. Oestergaard et al. [1] have described a method for magnetically induced chemical analysis based on the manipulation of reagent coated paramagnetic beads of $3 \mu \mathrm{m}$ diameter using permanent magnets. Solé et al. [2] have reviewed several of the advantages of beads over assays which use wall-bound analytes (e.g., antibodies) including immunoglobulin $\mathrm{G}$ (IgG). Further, magnetic microspheres offer a simple and adaptable method for the automation of relatively complex chemical procedures by allowing for the dispersion, capture, and concentration of bead-bound analytes [2].

The magnetization of paramagnetic microspheres increases linearly with magnetic field strength until a saturation value (where the induced dipole remains approximately constant). 
Upon removal of the externally applied field, the magnetization quickly reduces to zero with negligible hystersis [2]. The magnetization of spheres suspended in a solution quickly induces dipole-dipole interactions and spheres are well known to form chains oriented along the magnetic field. This effect is shown in Figure 1 for $1 \mu \mathrm{m}$ diameter spheres in a field of order $30 \mathrm{mT}$. Upon removal of the magnetic field, Brownian motion quickly disperses the spheres and there is no observable residual magnetism. Paramagnetic microspheres are widely available commercially in diameter sizes ranging from $20 \mathrm{~nm}$ to $1 \mathrm{~mm}$, and with varying percentages of magnetite content (12\% - 60\%). Typically, paramagnetic spheres are polymer spheres doped with iron-oxide crystals (magnetite). Modified surface groups (e.g., carboxylic acid or primary amine) may be linked to the outer bead surface for use in immunoassays [3].

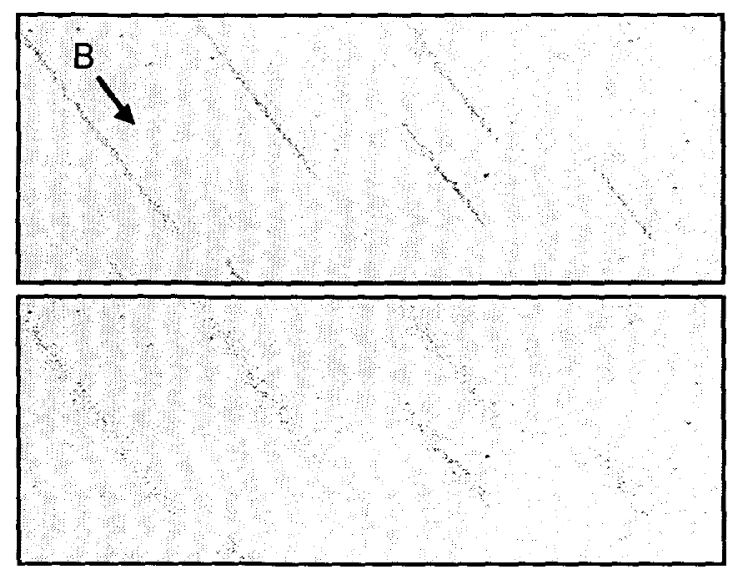

Figure 1. Chaining of paramagnetic microspheres. Top image shows linked sphere chains. When the magnetic field is removed, the spheres exhibit no residual magnetism and disperse within seconds due to Brownian motion (bottom image). Images were obtained using transmitted light microscopy with a $40 x$ objective.

Magnetic-based manipulation of beads in solution commonly relies on the use of permanent magnets to provide the external field. An alternate approach is to use a currentcarrying wire, such as a solenoid, to induce a controllable magnetic field based on the supplied current. A solenoid wrapped around the outer surface of a capillary generates a field predominantly oriented along the centerline axis of the capillary. This paper describes preliminary experiments using a solenoid-based bead manipulation device.

\section{Theory}

The magnetic force magnitude on a paramagnetic microsphere in solution is given by [1],

$$
\left|F_{m}\right|=\frac{1 \Delta \chi^{V}}{z}\left|\nabla B^{2}\right|
$$

For fields below the saturation value, the sphere-to-liquid magnetic susceptibility difference, $\Delta \chi$, can be thought of as a material constant, which is usually determined empirically. A single paramagnetic microsphere quickly reaches a terminal velocity, $v$, which can be expressed in terms of the field using the Stokes drag force, $\left|F_{D}\right|[4]$ :

$$
\left|F_{D}\right|=6 \pi R \eta|v|
$$

Equating this force with the magnetic force,

$$
|v|=\frac{1}{9 \mu_{0} \eta} \Delta \chi R^{2}\left|\nabla B^{2}\right|
$$

The microspheres also acquire a settling velocity, $v_{s}$, due to gravity which is calculated from a balance of drag, weight and buoyancy force [5]:

$$
v_{s}=\frac{1 \Delta \rho g d^{2}}{18}
$$

\section{Numerical Solenoid Field Model}

To characterize the magnetic field inside the solenoid, we created an axisymmetric magnetostatic solenoid model using Quickfield finite-element analysis software (TeraAnalysis) and solved for the radial and axial magnetic field profiles $\left(B_{r}(r, z)\right.$ and $B_{z}(r, z)$, respectively) for $r / r_{i}=0.0, r / r_{i}=0.5$, and $r / r_{i}$ $=1$.0. Input parameters used in the model (e.g., solenoid radius and current) are based on measured values of the solenoid properties used in experiments. The modeling results are shown in Figure 2. Although the radial magnetic field increases with radial distance, the total magnetic field remains largely axial. At the capillary wall $\left(r / r_{i}=1.0\right)$, we find that the axial component of the field within the solenoid is greater than $90 \%$ of the total field magnitude even in solenoid regions of increased magnetic field fringing. 

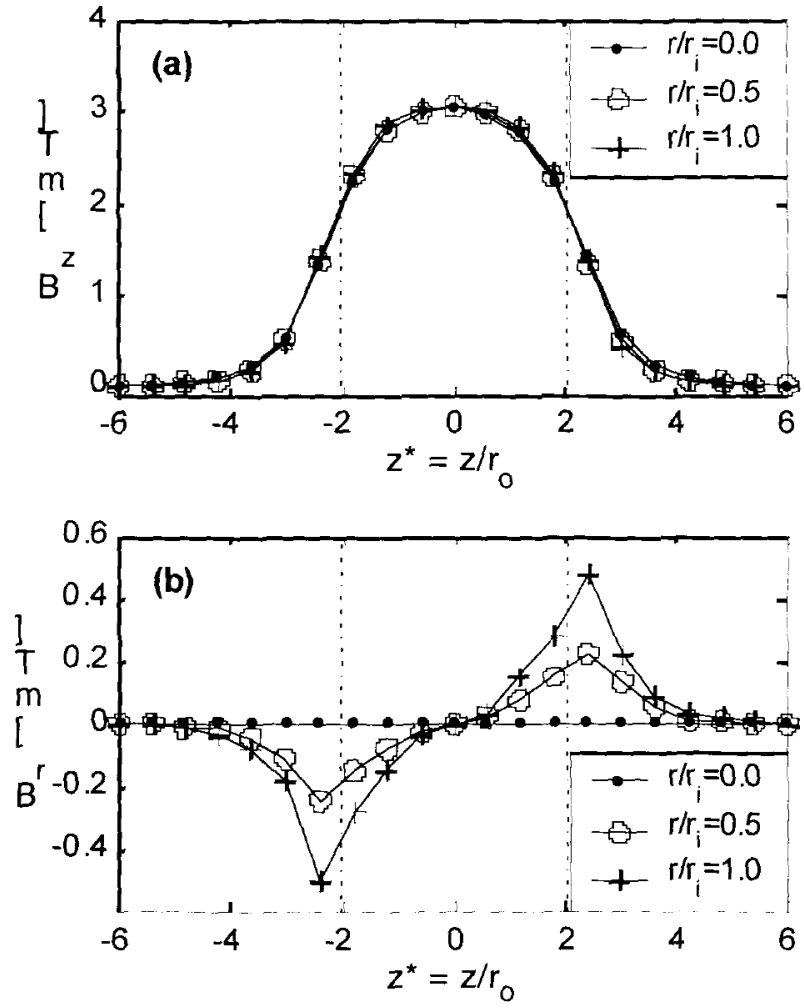

Figure 2. The axial magnetic field (a) and radial magnetic field (b) inside the solenoid, as determined by the solution to a Quickfield magnetostatic model of the solenoid. The solenoid lies between the two vertical dotted lines, from $z / r_{0}=-2.0$ and $z / r_{0}=+2.0$. Even though the radial magnetic field increases from $r / r_{i}=0.0$ to $r / r_{i}=1.0$, the axial field near the capillary wall remains nearly the same as the centerline axial field.

\section{EXPERIMENTAL}

We used epifluorescent microscopy to image the chaining and motion of paramagnetic spheres under flowing and stationary liquid conditions. Figure 3 shows a schematic of the imaging system including an inverted microscope configuration (Nikon) and a $4 \mathrm{~Hz} C \mathrm{CD}$ camera (Micromax $5 \mathrm{MHz}$, Roper Scientific). Illumination for the experiments was provided by a mercury $(\mathrm{Hg})$ lamp source. The excitation and emission of the microsphere fluorescence was $480 \mathrm{~nm}$ and $520 \mathrm{~nm}$, respectively. A 20x air objective with a numerical aperture of 0.45 was used.

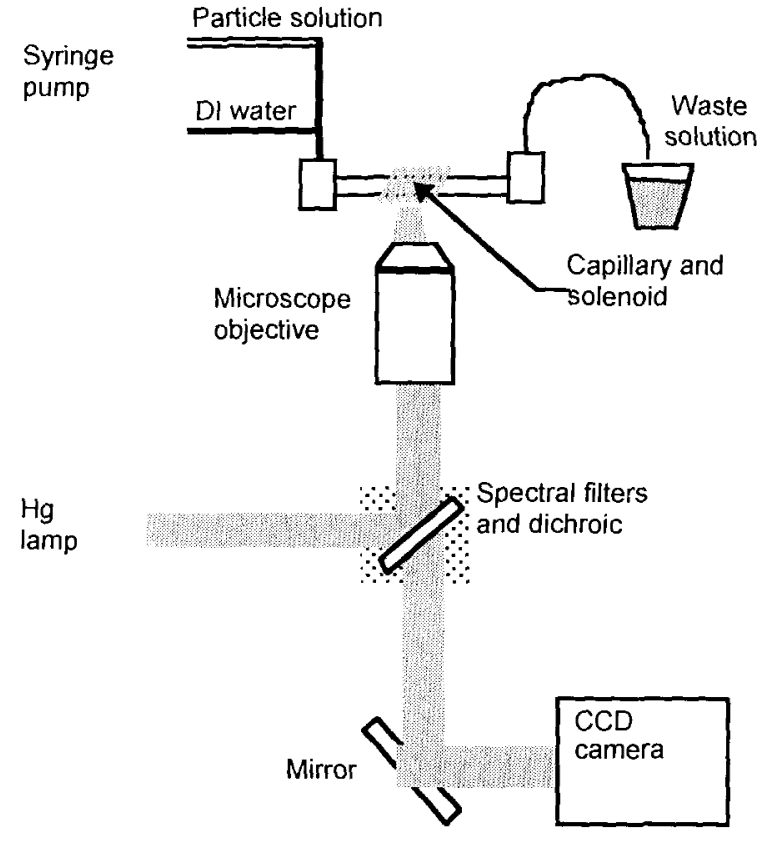

Figure 3. Schematic of experimental setup. An acrylic fixture holds the $1.3 \mathrm{~mm}$ borosilicate glass capillary, solenoid coil, and slide with silicone immersion oil.

We are in the process of evaluating the performance of several capillary solenoid designs, two of which are shown in Figure 4. The ultimate goal of the work is to apply a technology developed at the Lawrence Livermore National Laboratory (LLNL) for depositing copper films lithographically onto the outer surface of a cylindrical glass capillary. The solenoids are fabricated using a laser lathe apparatus where the spindle stage turns the workpiece (a $1.3 \mathrm{~mm}$ glass capillary) while a translation stage moves the piece along the axis of rotation. An argon-ion (Ar-ion) laser beam $(<5 \mathrm{~mW}$ power, multiline output with wavelengths between $330 \mathrm{~nm}$ and 366 $\mathrm{nm}$ ) is focused to a $5 \mu \mathrm{m}$ line using a cylindrical lens and a microscope objective to pattern the workpiece. The $1.3 \mathrm{~mm}$ glass capillary tube is first sputter-coated with a titanium (Ti) adhesion layer $(500 \AA)$, followed by a copper $(\mathrm{Cu})$ seed layer $(3000 \AA)$ using a rotation fixture to ensure uniform metal coverage. The $\mathrm{Ti} / \mathrm{Cu}$ multilayer provides a conductive surface for electroplating the conformal photoresist layer (Shipley's, PEPR 2400 positive electrodeposited photoresist). Next, a layer (8-12 $\mu \mathrm{m})$ of PEPR is coated conformally onto the glass capillary. The laser lathe apparatus is then used to pattern the solenoid strips onto the layers. After laser writing, the PEPR coating is developed, forming trenches down to the copper layer. The remaining PEPR coating is then used as a plating mask for the electrolytic deposition of $10 \mu \mathrm{m}$ of $\mathrm{Cu}$. The PEPR coating, the copper seed layer, and the titanium adhesion layer are removed with standard liquid etches, leaving a $10 \mu \mathrm{m}$ thick copper coil with $115 \mu \mathrm{m}$ wide lines and a pitch of $2^{\circ}$. On each side of the coil are $1 \mathrm{~mm}$ contact pads used for electrical 
connections. Each contact pad is connected to the coil by a 1 $\mathrm{mm}$ copper line.

As a preliminary effort, we are characterizing the performance of hand-wound coils of $100 \mu \mathrm{m}$ diameter. The visualization experiments performed with these hand-wound coils are presented in this paper. These coils were fabricated by hand-wrapping the $100 \mu \mathrm{m}$ coated copper wire around a 1.3 $\mathrm{mm}$ outer diameter $(0.7 \mathrm{~mm}$ inner diameter $)$, borosilicate glass capillary. The copper wire (Phelps Dodge, AWG 38) has a polyurethane insulation with a Nylon overcoat and an electrical resistance of $2 \Omega / \mathrm{m}$. To create ten evenly spaced windings over a total length of $2.7 \mathrm{~mm}$, each winding had a pitch of approximately $4^{\circ}$. The total resistance of the coil and its electrical leads was $2 \Omega$.

(a)

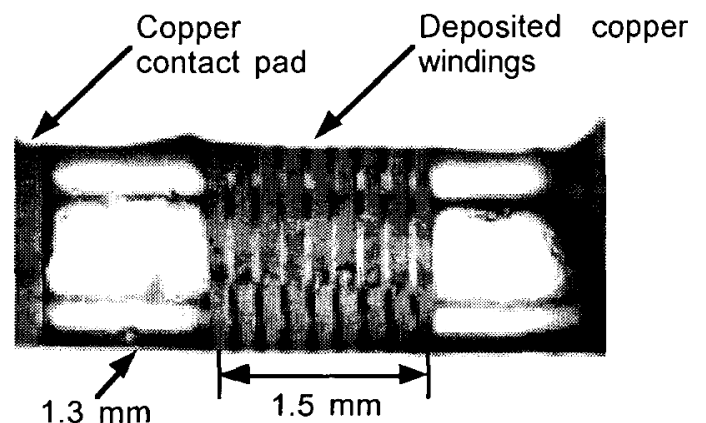

(b)

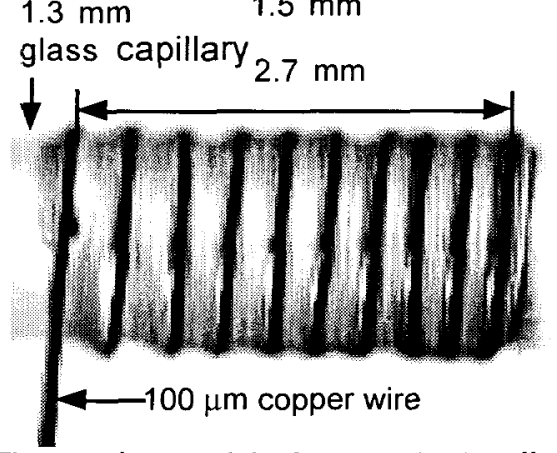

Figure 4. The top image (a) shows a test coil developed at the Lawrence Livermore National Laboratory (LLNL). There are nine copper windings deposited onto the outer surface of a $1.3 \mathrm{~mm}$ (outer diameter) glass capillary. Another test coil (b) was developed by winding $100 \mu \mathrm{m}$ (AWG 38) coated copper wire around the surface of a $1.3 \mathrm{~mm}$ (outer diameter) glass capillary. The wire coating is polyurethane with a Nylon overcoat. Ten windings are visible in the figure, with the overall solenoid length being $2.7 \mathrm{~mm}$.

An acrylic fixture to support individual test capillaries for flow and imaging studies was developed. Figure 5 shows this fixture. The ends of the glass capillary were inserted into holes drilled into the acrylic reservoir blocks located at the capillary inlet and outlet, respectively. The capillary was sealed to the fixture using a silicone rubber adhesive sealant. Holes were drilled and tapped on the top surfaces of the acrylic reservoir blocks to accommodate fittings connected to thin Teflon tubing (1.6 mm outer diameter). Flow through the tubing was delivered using a programmable dual-syringe pump (Harvard
Apparatus) that controlled $2 \mathrm{ml}$ borosilicate glass syringes (Popper and Sons) with an $8.9 \mathrm{~mm}$ internal diameter. To reduce optical distortion due to the curved glass capillary surface, silicone immersion oil (Olympus, $\mathrm{n}_{\mathrm{d}}=1.52$ ) was wicked between the outer glass capillary surface and a $1.3 \mathrm{~mm}$ thick glass microscope slide. The glass slide and microscope objective were separated by an air gap of approximately $5 \mathrm{~mm}$.

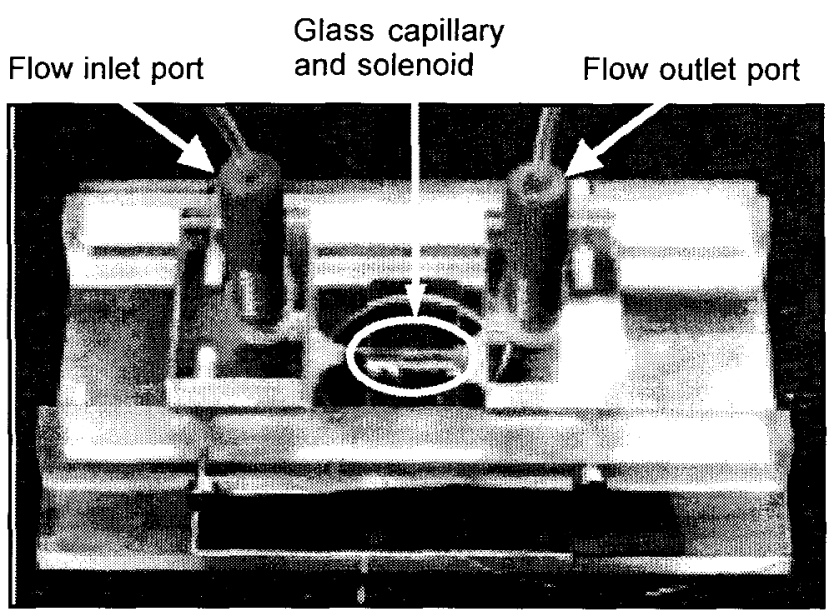

Figure 5. An acrylic test fixture was used to support the glass capillary with solenoid windings around its outer surface. The image shows the plastic tube fittings (Upchurch Scientific) and connecting Teflon tubing (1.6 mm outer diameter) for the inlet and outlet device ports.

The microbead solution consisted of streptavidin coated fluorescent paramagnetic microspheres (Bangs Laboratories), deionized (DI) water, and Triton X-100 surfactant (used only for the purpose of these visualizations). Prior to use, the vial of stock paramagnetic microspheres solution (46\% magnetite and $1 \%$ solids content by weight) was sonicated for 25 minutes to resuspend the microspheres $(960 \mathrm{~nm}$ diameter, $1.7 \mathrm{~g} / \mathrm{ml}$ density). Three drops of microsphere stock solution $(0.01 \mathrm{ml})$ were added to $7 \mathrm{ml}$ of DI water. Triton X-100 surfactant was added to the prepared solution ( $<0.1 \%$ by volume) in order to reduce adsorption of microspheres onto the glass channel walls. The final prepared solution was degassed using a vacuum pump for approximately 40 minutes in an effort to prevent exsolution of gases due to the elevated temperatures of the current-carrying coil.

\section{RESULTS AND DISCUSSION}

\section{Single Bead Magnetophoresis}

An example trajectory of a single paramagnetic microsphere subject to a magnetic field of $400 \mathrm{mT}$, a magnetic field gradient a $11 \mathrm{mT} / \mathrm{mm}$, and gravity is shown in Figure 6 . These field measurements were obtained using a Hall-effect probe (F. W. Bell Model 5080). The gravitational setting velocity, $v_{s}$, is 0.3 $\mu \mathrm{m} / \mathrm{s}$. 


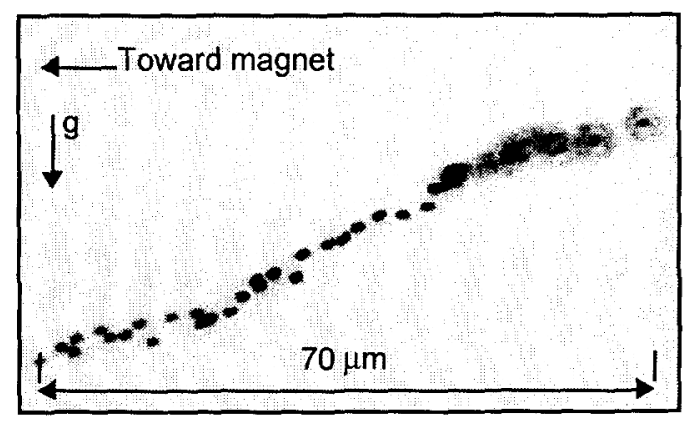

Figure 6. Magnetically induced drift for single microsphere. Image is a composite (sum) image formed from 40 successive frames captured with a 12-bit interline CCD camera (Roper Scientific). Epifluorescence microscopy was used with particle excitation and emission peak wavelengths of $480 \mathrm{~nm}$ and $520 \mathrm{~nm}$, respectively. The trajectory of a single microsphere toward the magnet face (located at the left of the field) is shown, with an approximate magnetic drift velocity of $1.8 \mu \mathrm{m} / \mathrm{s}$. The mean and standard deviation drift velocities computed from a sample set of $10 \mathrm{microspheres}$ were $1.1 \mu \mathrm{m} / \mathrm{s}$ and 0.7 $\mu \mathrm{m} / \mathrm{s}$, respectively.

\section{Capillary Thermal Response}

The relatively high current and finite resistance through both of our solenoid designs requires the consideration of thermal effects in the solenoid operation. At $0.75 \mathrm{~A}$, the wirewound solenoid coil dissipates less than $1 \mathrm{~W}$ of heat into the capillary and surrounding environment. This heat dissipation can lead to a significant temperature rise in the fluid inside the glass capillary and, depending on the thermal resistance to a heat sink, may cause boiling or, more commonly, force dissolved gases to emerge from solution. We have observed the latter at currents above $0.3 \mathrm{~A}$ for our setup and therefore outgas solutions prior to use as previously described. To approximately characterize the temperature of the fluid during operation of the coil, we obtained temperature measurements by placing a K-type thermocouple with a $700 \mu \mathrm{m}$ wire in contact with the fluid within the solenoid region of the capillary. Since the thermocouple wire increases the radial thermal conductivity of the capillary and water, these measurements are only an approximate measure of the running conditions. Temperature readings recorded every 5-10 seconds over a period of a few minutes are shown in Figure 7 for four current values.

For a current of $0.25 \mathrm{~A}$, the fluid temperature rise inside the coil is approximately $2{ }^{\circ} \mathrm{C}$ over a period of 30 seconds. For a current of $0.75 \mathrm{~A}$, the fluid temperature increases by $23^{\circ} \mathrm{C}$ in 30 seconds before reaching a steady-state temperature.

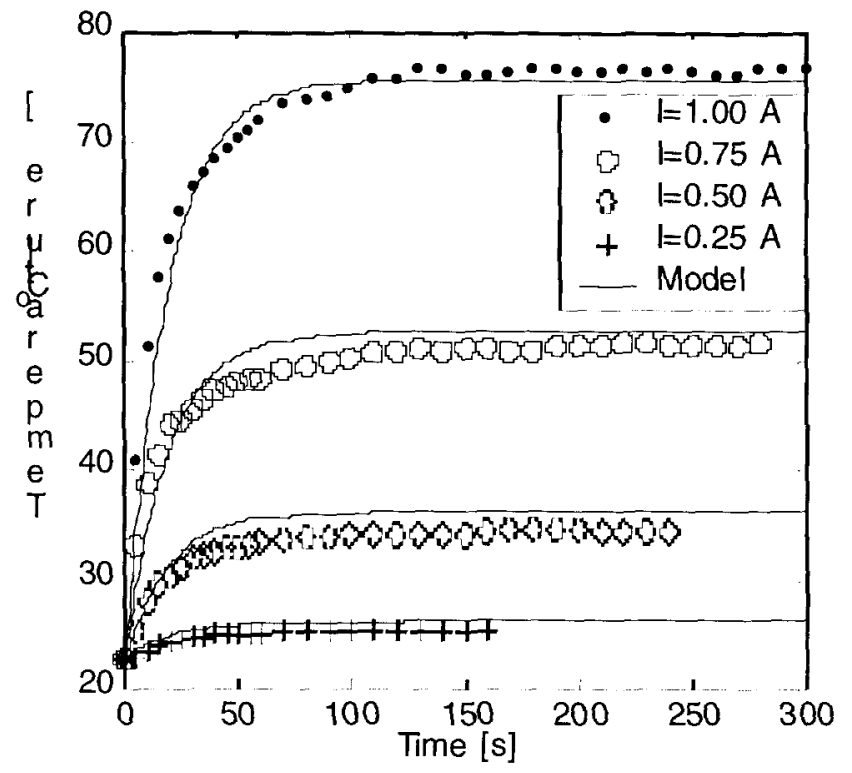

Figure 7. Solenoid thermal response. The fluid temperatures inside the wire solenoid over time, for currents of $0.25,0.50,0.75$ and $1.00 \mathrm{~A}$, measured by a $\mathrm{K}$ type nickel-chromium/nickel-aluminum thermocouple and Omega $\mathrm{HH} 22$ thermocouple microprocessor. These data were fit to an unsteady heat transfer model using an $h_{\text {eff }}$ value of $70 \mathrm{~W} /\left(\mathrm{m}^{2} \mathrm{~K}\right)$ in all cases (solid black curves). While the temperature at $0.25 \mathrm{~A}$ current increased only 3 ${ }^{\circ} \mathrm{C}$, at $1.00 \mathrm{~A}$ of current, the temperature increased by 50 ${ }^{\circ} \mathrm{C}$.

The measured solenoid thermal response is consistent with an unsteady heat transfer model for an infinite cylinder with an approximately constant heat transfer coefficient [6]. With an

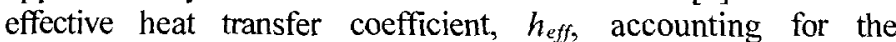
immersion oil and ambient air, and heat generation due to the Joule dissipation from the solenoid current, the model predicts a first order exponential temperature curve similar to the measured data in Figure 7. After fitting the model to the data using $h_{\text {eff }}$ as a fitting parameter, we found that an $h_{\text {eff }}$ value of 70 $\mathrm{W} /\left(\mathrm{m}^{2} \mathrm{~K}\right)$ yields a similar solenoid thermal response for each of the four tested currents.

The temperature measurements shown in Figure 7 show that the performance of the uncooled solenoid capillary as a bead trapping or retention device will be limited by the maximum allowable temperature rise of the assay. Assuming a constant effective convective thermal coupling to the environment, the steady state temperature rise is related to the heat dissipation of the coil as,

$$
\Delta T \sim \frac{I^{2}}{L^{2}}
$$


The symbol ' $\sim$ ' is used here to indicate a direct proportionality relation. For an ideal solenoid, the magnetic field strength is related to the applied current [7]:

$$
B=\frac{\mu_{0} I N}{L}
$$

Further, a one-dimensional simplification of Equation 1 allows for the approximation of the magnetic force on a particle as being,

$$
F_{m} \sim \frac{B^{2}}{L}
$$

Therefore, the magnetic force on a paramagnetic bead (and hence, its terminal velocity) for a given temperature-limited magnetic field is expected to be relatively insensitive to the geometry of the coil:

$$
F_{m} \sim v \sim \Delta T
$$

One method of reducing the operating temperature of the coils is to decrease the resistance of the solenoid windings. A possible approach is to double the cross-sectional area of the wire and decrease the pitch of the windings. Such modifications should reduce the Joule heating of the coil while keeping field and field gradients constant. We are currently in the process of redesigning the experiment to mitigate the effects of temperature.

\section{Chain Formation and Retention}

We performed a set of experiments of chain formation and retention (under flowing conditions) of bead chains. The prepared particle solution was driven into the glass capillary at a flow rate of $1 \mathrm{ml} / \mathrm{min}$ until a steady fluid stream was established in order to fill the capillary in a repeatable fashion. The syringe pump was then deactivated, stopping the flow. We subsequently performed the investigations described below.

First, we observed the chaining process as a function of the DC current through the wire-wound solenoid. Here we present the results for a DC solenoid current of 0.75 A (3 mT). Successive images were collected at $0.1 \mathrm{~Hz}$ with an exposure time of $10 \mathrm{~ms}$ during the chaining process. The imaging sequence procedure was repeated several times, moving the field of view through several regions throughout the solenoid (e.g., solenoid ends and center). New particle solution was flowed into the test capillary region before each new imaging sequence. The intensity of the images was inverted so that dark dots and streaks represent high intensity particle and particle chain images, respectively. The vertical shade regions in the image correspond to three observable passes of the solenoid coil. As expected, the rate of the chaining event was observed to increase with magnetic field strength, as well as microsphere seed density in a given flow. The image sequence shown in Figure 8 shows the evolution of chain formation near the center of the wire solenoid carrying $0.75 \mathrm{~A}$.

In the absence of flow, paramagnetic chains are most densely collected near the center of the solenoid, near the $5^{\text {th }}$ and $6^{\text {th }}$ coil turns of the 10-turn coil. In the high chain density region of the solenoid, adjacent chains combine to form packed, multi-level chain structures. Such multi-level chains are not prominent in solenoid coil regions near the ends $\left(1^{\text {st }}\right.$ or $10^{\text {th }}$ coils). Throughout the axis of the solenoid, chains do not undergo axial translation, and chain motion seems to be induced only by inter-chain attraction. Paramagnetic microsphere chains also collect most prominently near the bottom capillary wall due to gravitational settling. This observation was made by optically sampling the vertical space of the capillary by traversing the microscope depth of field at 10 $\mu \mathrm{m}$ increments. Upon magnetic field deactivation, the microsphere chains readily break apart. However, individual particles often adhere to the bottom glass capillary wall during the experiments.

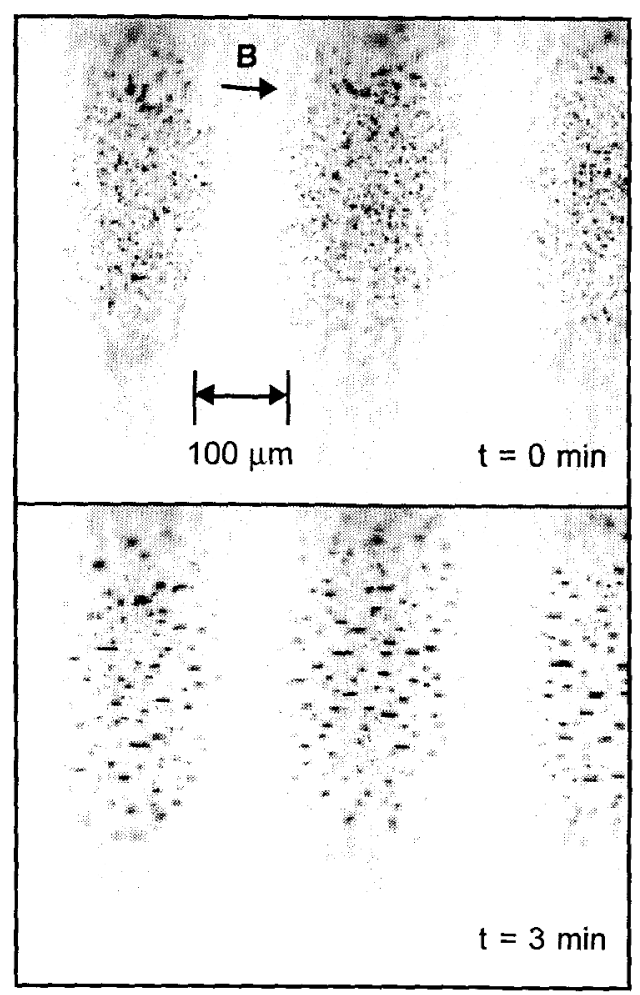




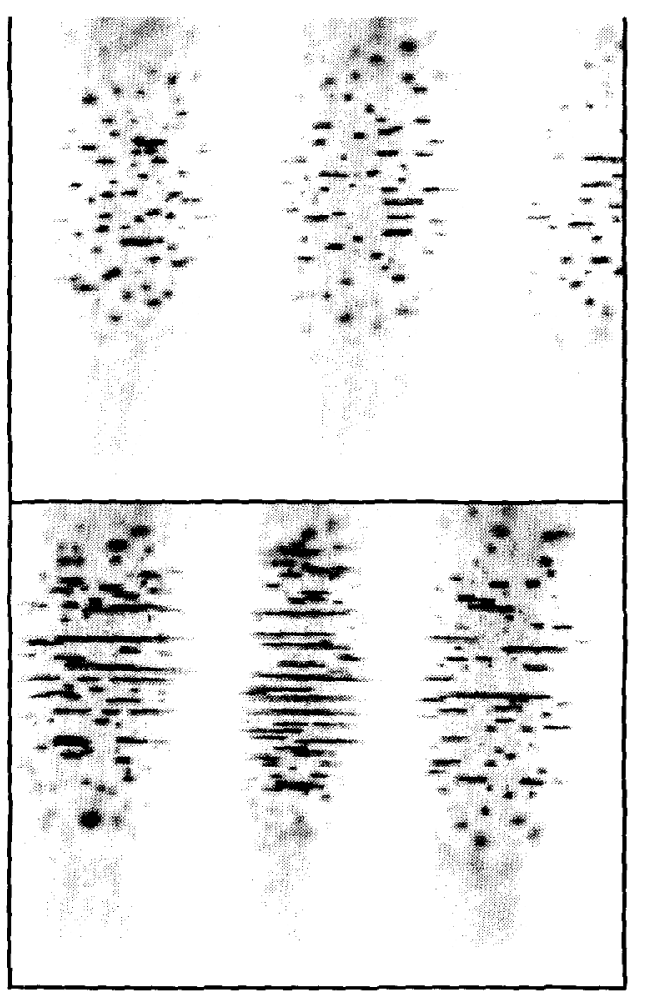

Figure 8. Chain formation within center region of handwound solenoid over time (left column). The image sequence shows $0.96 \mu \mathrm{m}$ paramagnetic microspheres linking to form chains oriented along the induced magnetic field direction. The current through the solenoid was 0.75 $A$, and the field strength was approximately $3 \mathrm{mT}$. After 12 minutes, the formed chains were approximately $200 \mu \mathrm{m}$ long.

A second set of experiments was conducted to estimate the maximum possible flow rate through the capillary without dislodging already-formed paramagnetic bead chains subject to an applied magnetic field. Again, chains were formed at a 0.75 A solenoid current for $12-15$ minutes. DI water was then flowed through the capillary using a syringe pump at increasing flow rates beginning with $0.01 \mathrm{ml} / \mathrm{min}$ and increasing by 0.006 $\mathrm{ml} / \mathrm{min}$ increments until we observed the chained paramagnetic particles begin to flow out of the coil. The CCD camera was used to capture the motion of the bead chains exiting the solenoid from the rightmost coil, as shown in Figure 9. The top and bottom regions of the image field are out of focus due to optical distortion resulting from the curvature of the cylindrical capillary surface.

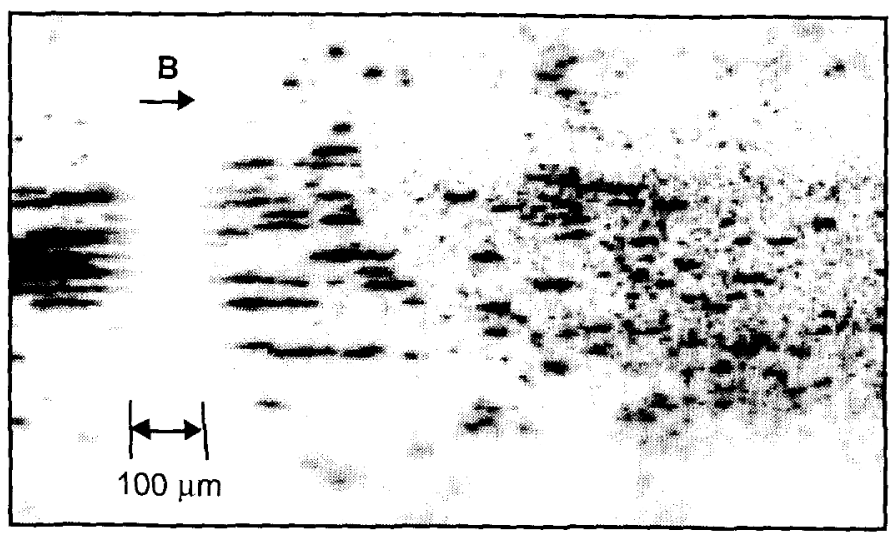

Figure 9. Paramagnetic particle chains dispersing near the exit of the capillary solenoid. DI water flows from left to right through the capillary at $0.02 \mathrm{ml} / \mathrm{min}$ (bulk flow speed $850 \mathrm{~m} / \mathrm{s})$. The solenoid was operated at 0.75 A current, and chains were allowed to form for approximately 15 minutes before initiating flow.

Flow rates greater than $0.02 \mathrm{ml} / \mathrm{min}$ force the chains to exit the coil region. The first chains to leave the coil are, as expected, near the center of the fluid stream, where the fluid velocity is approximately $1700 \mu \mathrm{m} / \mathrm{s}$, assuming a fully developed Poiseuille flow profile. Chains located approximately $10 \mu \mathrm{m}$ from the bottom annular periphery experience a flow velocity magnitude that is approximately $5 \%$ of the centerline value, or $90 \mu \mathrm{m} / \mathrm{s}$.

As the chains and particles flow downstream through the rapidly decaying magnetic field near the edge of the solenoid, the paramagnetic particles lose their magnetization and disperse.

\section{CONCLUSIONS}

A sub-millimeter solenoid device has been developed to assist the evaluation of compact magnetic-based separation devices that operate without permanent magnets. Preliminary experiments using a hand-wound coil of $100 \mu \mathrm{m}$ diameter wrapped around a $1.3 \mathrm{~mm}$ glass capillary demonstrate that the formation and retention of paramagnetic microsphere chains is possible under a supplied DC current of $0.75 \mathrm{~A}$. At $0.75 \mathrm{~A}$, the solution within the capillary is heated by approximately 30 ${ }^{\circ} \mathrm{C}$ within 2 minutes. The induced magnetic field within the solenoid is predominantly along the main axis of the capillary and has a magnitude of approximately $3 \mathrm{mT}$. Under these conditions, $200 \mu \mathrm{m}$ bead chains form along the field lines within 12 minutes. The highest chain concentration occurs at the center region of the solenoid coil windings, near the bottom capillary wall. Such chains are retained in place against a bulk flow velocity of $850 \mu \mathrm{m} / \mathrm{s}$.

Future work will further address heat transfer effects in current-carrying solenoid devices. We will attempt to minimize net heat input to the capillary solution. Additionally, we will use the solenoid device to form and retain chains in the presence of a flowed linker solution (e.g., glutaraldehyde) to create permanently-bound chains that will be 
released from the solenoid trap upon field deactivation. The binding of paramagnetic bead chains has been demonstrated by Furst et al. [8].

\section{ACKNOWLEGMENTS}

This work is financially supported by the National Science Foundation (NSF) under Contract No. CTS-9980860. Financial support for Mr. L. Garcia is provided in part by a grant from The Paul and Daisy Soros Fellowships for New Americans. Financial support for Mr. L. Cheung is provided by a National Defense Science and Engineering Graduate (NDSEG) Fellowship. Portions of this work were performed under the auspices of the U.S. Department of Energy by the University of California, Lawrence Livermore National Laboratory under Contract No. W-7405-Eng-48.

\section{REFERENCES}

1. Ostergaard, S., Blankenstein G., Dirac, H., and Leistiko, O., "A novel approach to the automation of clinical chemistry by the controlled manipulation of magnetic particles", Journal of Magnetism and Magnetic Materials, 194 156-162 (1999).

2. Solé, S., Merkoci A., Alegret, S., "New materials for electrochemical sensing III. Beads", Trends in Analytical Chemistry, 20 (2) 102-110 (2001).

3. Bangs Laboratories, "TechNote \#102, Magnetic Microspheres", (1999).

4. White, F., Viscous Fluid Flow, $2^{\text {nd }}$ edition, McGrawHill, Inc., New York (1991).

5. Pai, V., Chen, C., and Haik, Y., "Microscopic flow visualization system for fluids in magnetic field", Journal of Magnetism and Magnetic Materials, 194 262-266 (1999).

6. Eckert, E., and Drake, R., Analysis of Heat and Mass Transfer, McGraw-Hill, Inc., New York (1972).

7. Griffiths, D., Introduction to Electrodynamics, $2^{\text {nd }}$ edition, Prentice Hall, New Jersey (1989).

8. Furst, E., Suzuki, C., Fermigier, M., Gast, A., "Permanently Linked Monodisperse Paramagnetic Chains", Langmuir, 14 7334-7336 (1998). 